\title{
Vers un naturalisme rural en Autriche : Courbet, Zola et Ferdinand von Saar
}

Zu einem ländlichen Naturalismus in Österreich: Courbet, Zola, Ferdinand

von Saar

Toward Austrian rural naturalism: Courbet, Zola, Ferdinand von Saar

\section{Carolyn Snipes Hoyt}

\section{OpenEdition}

\section{Journals}

Édition électronique

URL : http://journals.openedition.org/austriaca/524

DOI : 10.4000/austriaca.524

ISSN : 2729-0603

\section{Éditeur}

Presses universitaires de Rouen et du Havre

\section{Édition imprimée}

Date de publication : 1 juin 2018

Pagination : 55-69

ISBN : 979-10-240-1233-9

ISSN : 0396-4590

\section{Référence électronique}

Carolyn Snipes Hoyt, « Vers un naturalisme rural en Autriche : Courbet, Zola et Ferdinand von Saar », Austriaca [En ligne], 86 | 2018, mis en ligne le 01 juillet 2020, consulté le 30 janvier 2021. URL : http:// journals.openedition.org/austriaca/524 ; DOI : https://doi.org/10.4000/austriaca.524 


\section{Vers un naturalisme rural en Autriche: Courbet, Zola et Ferdinand von Saar}

À peu près en même temps que Zola rédigeait Les Rougon-Macquart (1869-1893), Ferdinand von Saar était en train de créer une série de nouvelles dépeignant la vie en Autriche, dont quatorze ont été publiées entre $1866^{1}$, juste après la guerre austro-prussienne et la défaite des Autrichiens à Königgrätz, et 1892. Viennois lui-même, avec des ancêtres qui avaient servi l'Empire autrichien et reçu ainsi la particule nobiliaire von précédant leur nom de famille, Saar avait grandi comme fils unique d'une veuve qui fut obligée de retourner avec son enfant chez son père; à la mort de ce dernier, elle dut se débrouiller seule pour elle et son fils ${ }^{2}$.

Immédiatement après avoir fini ses études secondaires, Saar entra dans l'armée et pendant son service il eut l'avantage de faire l'expérience d'une variété de milieux, de paysages et de peuples, parmi lesquels plusieurs sont devenus les sujets de ses écrits en prose - surtout le milieu militaire, la haute société viennoise, mais aussi les marginaux ${ }^{3}$. Tandis que Saar aspirait en vain à devenir un dramaturge de renom, il avait connu un certain succès comme poète, au moment où sa nouvelle Die Steinklopfer (Les Casseurs de pierre) parut, en 1873-1874 ${ }^{4}$. Cet ouvrage dépeint le sort difficile des ouvriers qui cassaient les pierres dans un paysage montagnard près de Vienne, pour construire la célèbre

1. Karl Wagner, «(Anti-) Naturalism in Austrian Literature», Excavatio, vol. 15, 2001, p. 224.

2. Herbert Klauser, Ein Poët aus Österreich : Ferdinand von Saar - Leben und Werk, Wien, Literas-Universitätsverlag, 1990, p. 9-10.

3. Ibid., p. 14 et 17.

4. Rainer Baasner, «Happy-End trotz Schopenhauer oder Das Glück eines armen Soldaten. Ferdinand von Saars Die Steinklopfer ", dans Karl Konrad Polheim (dir.), Ferdinand von Saar. Ein Wegbereiter der literarischen Moderne. Festschrift zum 150. Geburtstag, Bonn, Bouvier, 1985, p. 51, note 1. 
ligne de chemin de fer du Semmering, terminée en $1854^{5}$. Afin de s'excuser auprès de sa protectrice aristocrate, Marie zu Hohenlohe, pour sa prose simple et ses personnages peu compliqués, Saar avait expliqué que l'époque demandait justement un traitement réaliste:

[...] aber der Dichter, wenn auch als solcher geboren, wird doch auch durch das Leben und durch seine Zeit bedingt, welch letztere nun einmal eine realistische ist. So viel Idealismus glaube ich mir aber doch noch bewahrt zu haben, daß ich nicht zum platten Naturalismus herabsinke - obgleich ich in meiner dritten Novelle "Die Troglodytin" im künstlerischen Realismus bis zum äußersten gehe 6 .

En fait, on considère Die Steinklopfer comme un des premiers ouvrages en prose dans la littérature de langue allemande qui traitent du prolétaire ${ }^{7}$; et Die Troglodytin (1889), qu'on examinera plus loin dans cette étude, aurait subi l'influence du roman naturaliste rural de Zola, La Terre ${ }^{8}$. Il faut d'ailleurs tenir compte du fait que certains autres genres dans la littérature populaire germanophone - le conte de fées, par exemple - mettent également, selon Katra Byram, l'accent sur la pauvreté et la misère des gens ruraux et sur la désintégration du tissu social ${ }^{9}$. Pendant cette période, les pièces de théâtre très populaires de Ludwig Anzengruber sur le prolétariat rural en Autriche s'attiraient la menace de censure. Deux ans avant la parution de la nouvelle de Saar, Anzengruber put enregistrer son premier succès, avec la première à Vienne de sa pièce Die Kreuzelschreiber, dans laquelle, curieusement, un Steinklopferhans

5. Construite entre 1848 et 1854 , la ligne de chemin de fer du Semmering, avec ses tunnels et viaducs, est la première ligne de montagne à voie normale en Europe. Un millier d'ouvriers sont morts (accidents et épidémie) pendant sa construction.

6. Saar à Fürstin Marie zu Hohenlohe, 24 septembre 1887. Cité dans Karl Wagner, art. cité, p. 224-225. En français : « [...] mais l'écrivain - même s'il est né pour écrire se trouve marqué néanmoins par sa vie et son époque, et la nôtre est justement réaliste. Néanmoins, je pense avoir gardé assez d'idéalisme pour éviter une chute dans le naturalisme plat - bien que dans ma troisième nouvelle "Die Troglodytin" je pousse le réalisme jusqu’à ses limites. »Toutes les traductions de l'allemand en français sont les miennes. Il est question ici de la $2^{\mathrm{e}}$ publication de Die Steinklopfer, cette fois-ci dans la collection Novellen aus Österreich, publiée en 1877.

7. Herbert Klauser, op. cit., p. 103.

8. Karl Wagner, art. cité, p. 224.

9. Katra A. Byram, "Fairy Tales in the Modern(ist) World: Gerhart Hauptmann's Bahnwärter Thiel and Marie von Ebner-Eschenbach's Das Gemeindekind », The German Quarterly, vol. 86, $\mathrm{n}^{\circ}$ 2, printemps 2013, p. 142. L'auteur remarque que les problèmes du conte de fées résonnent avec ceux qui occuperaient le naturalisme. 
joue un rôle capital ${ }^{10}$. Comme représentation des couches sociales des campagnes, c'est la nouvelle Die Steinklopfer de Saar qui représente, dans la littérature autrichienne, un premier pas vers un naturalisme rural en prose. Selon Klauser, ce ne fut d'ailleurs pas exactement l'intention de l'auteur, qui avait voulu simplement montrer sa solidarité avec la vie difficile de l'ouvrier: "Die Novelle „Die Steinklopfer,“ die mit Unrecht manchmal als Beginn der sozialen Arbeiterliteratur bezeichnet wird, ist reine Mitleidsdichtung ${ }^{11}$.» À ce sujet, Klauser estime que ce que dit le narrateur des Steinklopfer est révélateur de l'intention de l'auteur : "nur um zu zeigen wie Leid und Lust jedes Menschenherz bewegen und daß sich überall im kleinen abspielt die große Tragödie der Welt ${ }^{12}$.» En fait, Saar dédia la nouvelle à Josephine von Wertheimstein, une femme qui avait tant fait pour les pauvres. Wagner note d'ailleurs avec raison que la référence au célèbre tableau de Courbet du même titre et le choix de casseurs de pierre comme protagonistes, nous donnent un indice de l'intention de Saar de rattacher délibérément son récit au courant « réaliste ${ }^{13}$ ». En général, la prose en Autriche s'est tournée, à la fin du $\mathrm{XIX}^{\mathrm{e}}$ siècle, vers d'autres préoccupations - surtout vers l'individu et le psychologique, des courants qu'on peut déjà déceler dans les nouvelles de Ferdinand von Saar ${ }^{14}$.

10. Voir Manfred Kuhne, «Einleitung» et notes, dans Anzengrubers Werke in Zwei Bänden, Berlin-Weimar, Anbau-Verlag, 1971, t. I, p. 345. Nous reviendrons sur cette question plus loin.

11. Herbert Klauser, op. cit., p. 47 : «La nouvelle "Die Steinklopfer", qu'on identifie à tort parfois comme le commencement de la littérature ouvrière socialiste, est une littérature de pure compassion.»

12. Ferdinand von Saar, Die Steinklopfer, dans Novellen aus Österreich, Karl Wagner (éd.), Wien, Franz Deuticke, 1998, t. I, p. 88 : « seulement afin de montrer comment le chagrin et le désir agissent sur chaque cour humain et que partout la grande tragédie du monde a lieu en miniature. » Toutes les références aux deux nouvelles de Saar renvoient à cette édition en 2 tomes ou au «Nachwort» de Karl Wagner.

13. Karl Wagner, dans Novellen aus Österreich, op. cit., t. II, p. 307.

14. Pendant cette période, certains auteurs, comme Peter Rosegger, Ludwig Anzengruber et Marie von Ebner-Eschenbach, décrivaient la vie rurale en Autriche dans des ouvrages en prose, le plus souvent suivant les courants qu'on considérait comme "anti-moderne" et "anti-naturaliste", Heimatkunst ou Provinzkunst. Certains de ces auteurs, en particulier Saar et Rosegger, avaient réagi, dans un premier temps, de manière assez négative contre Zola et le naturalisme, mais, plus tard, ils commencèrent à admirer son œuvre et à adopter une partie de ses techniques (voir Karl Wagner, art. cité, p. 217-218 et 220-221). D'autres influences naturalistes sur la littérature autrichienne à cette époque, négative dans le cas de Saar, venaient de Gerhart Hauptmann et de Henrik Ibsen. Bahnwärter Thiel, la célèbre nouvelle naturaliste de Hauptmann, où il est question d'un cheminot, parut 
Au milieu du XIX ${ }^{\mathrm{e}}$ siècle, la figure du casseur de pierre servit de topos pour l'empiétement de l'industrialisation sur les campagnes, offrant l'occasion aux artistes et aux écrivains de sympathiser avec le prolétariat non urbain. Les casseurs de pierre (souvent des esclaves) avaient déjà été présents dans le paysage champêtre depuis l'antiquité. $\mathrm{Au} \mathrm{XIx}{ }^{\mathrm{e}}$ siècle, leur représentation, en tant que prolétariat rural, prit une signification particulière en rapport avec l'industrialisation qui transformait la société ${ }^{15}$. À la suite des révolutions de 1848 , le demisiècle devint le foyer de la convergence des discours sur le «roturier» post-révolutionnaire.

Le célèbre tableau de Gustave Courbet, intitulé Les Casseurs de pierre, peint et exposé en 1849, aurait bien pu servir d'inspiration à Saar. D'abord montré dans une exposition itinérante à Dijon, puis dans le salon parisien très ouvert de 1850-1851, il fit scandale, selon ses critiques, par sa sobre représentation, au premier plan et à grandeur nature, de ruraux ordinaires ${ }^{16}$. Manuel Jover explique que Courbet ne fut ni le premier ni le seul à dépeindre les classes ouvrières, mais, contrairement aux peintres réalistes du Second Empire, il rejeta l'anecdote et la mièvrerie en faveur d'une représentation sans pitié des faits ${ }^{17}$. Malgré l'immédiateté et la nouveauté de la représentation de ces deux ouvriers ${ }^{18}$, Émile Zola avait reproché à Proudhon ses louanges de Courbet comme «peintre socialiste» en raison de son choix de sujet, car le chef de file

en 1888. Dans sa correspondance avec Marie von Ebner-Eschenbach, au sujet des questions littéraires et artistiques, Saar manifesta son dégoût du naturalisme, expliquant qu'il était "Ibsen- und Hauptmannmüde» (fatigué d'Ibsen et de Hauptmann, cité dans Herbert Klauser, op. cit., p. 231). Saar avait une attitude plus positive envers Tourguenev, dont l'influence directe pourrait être repérée dans certaines nouvelles (ibid., p. 95).

15. Certes, le personnage de Victor Hugo, Jean Valjean, dans Les Misérables (1862), avait passé son temps en prison à casser des pierres. Hugo avait commencé la rédaction de ce roman avant la révolution de 1848 .

16. Voir Max Buchon, "Avertissement» [sur l'exposition de Courbet à Dijon, 1850], Le Peuple. Journal de la révolution sociale, 7 juin 1850, cité dans Timothy J. Clark, Image of the People. Gustave Courbet and the 1848 Revolution, London, Thames and Hudson, 1973, p. 162, "Appendix I». Ce tableau fut détruit en 1945, lors du bombardement de Dresde.

17. Manuel Jover, Courbet, Paris, Terrail, 2007, p. 53.

18. Vers la fin de 1849 , Courbet avait écrit une lettre, pendant un séjour à Ornans, sa ville natale, décrivant son dernier ouvrage. Il avait vu deux hommes en train de tailler des pierres à côté de la route et il les avait invités à venir dans son atelier. Voir Michael Fried, "Painter into Painting: On Courbet's "After Dinner at Ornans" and "Stonebreakers" », Critical Inquiry, vol. 8, n 4, été 1982, p. 634-636. 
de l'école naturaliste avait mis en avant la créativité de l'artiste en tant qu'individu $^{19}$.

Peu après, en 1851, Alphonse de Lamartine publia sa nouvelle Le Tailleur de pierres de Saint-Point, l'histoire fictive et hautement romancée d'un ouvrier très simple représenté comme brillant philosophe ${ }^{20}$. Saar déclara qu'il avait refusé de lire, pendant la rédaction de ses Steinklopfer, la traduction en allemand de cet ouvrage par Marie zu Hohenlohe; mais, plus tard, après avoir lu La Terre de Zola ${ }^{21}$, il fait cette remarque à la traductrice :

Wenn man "La Terre" von Zola noch in frischester Erinnerung hat, muß einem diese ideale ländliche Erzählung etwas fremdartig vorkommen. Welch ein Unterschied zwischen dem geradezu furchtbaren Realismus Zolas - und der gotterfüllten evangelischen Seele Lamartine ${ }^{22}$ !

En créant son couple de casseurs de pierre, Saar avait évité l'idéalisation exagérée du simple ouvrier qu'on remarque chez Lamartine, car ses personnages sont des gens ordinaires sans prétentions, quoique solides sur le plan éthique. Tout de même, Saar adoucit «le réalisme terrible» qu'il attribua à Zola, en imaginant un happy end de conte de fées - un sursis, car le sort de l'être humain change toujours, mais descend inévitablement vers le bas. C'est-à-dire que Saar et Zola souscrivaient à la philosophie pessimiste schopenhauerienne, du moins à un certain

19. Émile Zola, "Proudhon et Courbet», dans Écrits sur l'art, Jean-Pierre LeducAdine (éd.), Paris, Gallimard, 1991, p. 50-52. En ce qui concerne l'exposition d'ouvrages de Courbet en Autriche, voir Christian Huemer, "Une exposition (in)complète": Courbet in Vienna, 1873", Nineteenth-Century Art Worldwide, vol. 11, n ${ }^{\circ}$ 200, été 2012, http :/www.19thc-artworldwide.org/summer12/christianhuemer-courbet-in-vienna. La controverse autour du désir contrarié de Courbet d'exposer ses ouvrages à l'Exposition universelle à Vienne en 1873 n'avait pas trouvé d'écho dans le grand journal libéral Neue Freie Presse, qui n'a pas non plus parlé de l'exposition indépendante organisée par l'Österreichischer Kunstverein, où, selon Huemer, certains de ses tableaux furent exposés, mais pas Les Casseurs de pierre. C'était juste avant la parution de la nouvelle de Saar.

20. Au sujet du casseur de pierre comme figure symbolique du prolétariat pendant cette période révolutionnaire, Herbert Klauser mentionne, lui aussi, le groupe Courbet, Lamartine, Anzengruber et Saar (op. cit., p. 104).

21. La traduction en allemand de La Terre fut censurée en Autriche en 1888, une année après sa publication en France (voir Karl Wagner, art. cité, p. 218).

22. Saar à Hohenlohe, 9 janvier 1888. Cité dans Karl Wagner, art. cité, p. 225: "Quand on a encore à l'esprit le souvenir vivant de "La Terre" de Zola, ce conte représentant la vie idéale à la campagne semble forcément étrange. Quelle différence entre le terrible réalisme de Zola et l'âme ardente et pleine de Dieu de Lamartine! » 
moment ${ }^{23}$. Notons que, dans La Terre (1887), on trouve également la mention du métier de casseur de pierre. Non sans ironie, on surnomme Jacqueline, une jeune femme aux mœurs légères, «La Cognette »; c'est elle, la fille à "Cognet ", " ce soûlard qu'on voyait depuis vingt ans casser les cailloux sur la route ». Dans le même roman, le père Fouan s'exclame que, plutôt que de rester plus longtemps chez sa fille hargneuse et son mari, il «aimerai $[t]$ mieux casser les pierres sur la route ${ }^{24} »$. La Terre de Zola traite, d'ailleurs, de paysans libres, tandis que la nouvelle de Saar montre le prolétariat rural.

Par ailleurs, en 188o, six ans après la première parution des Steinklopfer, Anzengruber publia Geschichten vom Steinklopferhans / Die Märchen des Steinklopferhanns - une série d'histoires racontées en dialecte par le casseur de pierres qui paraît dans sa pièce Die Kreuzelschreiber ${ }^{25}$. Le livre s'ouvre par une mise en scène de forêts et de champs de blé qui se parlent, ce qui fait penser à l'environnement rural enchanté du conte de fées. Dans ce texte, l'animation de la nature prépare la narration de contes parodiant certaines coutumes du peuple et leurs croyances superstitieuses et/ou chrétiennes, mais annonçant aussi l'arrivée dans le paysage rural du nouveau monstre technologique - le train.

Le narrateur de Die Steinklopfer décrit, lui aussi, tout d'abord les forêts vierges près de Vienne, mais elles sont maintenant en transformation, envahies par des ouvriers, leurs habitations temporaires, leur saleté, le bruit de leur marteaux, leurs cris. Ici, peureux et silencieux, les animaux de la forêt observent tout ce qui se passe ${ }^{26}$. La nouvelle de Saar se situe entre la littérature traditionnelle populaire, comme le conte de fée et le « roman ${ }^{27}$ » ou l'hagiographie, et la littérature réaliste/naturaliste. On y remarque certes les procédés du conte de fée, dans la symbolique des couleurs (le bleu et le jaune) et des chiffres (surtout "quatre»), et dans le choix des motifs, comme la mention de fleurs et d'abeilles signifiant le bonheur. Mais l'intrigue de cette nouvelle s'apparente aussi à celle du mythe ou du «roman» - quand on considère, en son centre, le conflit

23. Voir David Baguley, Naturalist Fiction. The Entropic Vision, Cambridge, Cambridge University Press, 1990, p. 131, ainsi que Herbert Klauser, op. cit., p. 155 et Rainer Baasner, art. cité, p. 51-73.

24. Émile Zola, La Terre, Armand Lanoux (préf.), Paris, Le Livre de poche, 1979, p. 91 et 283 .

25. Plusieurs de ces contes avaient été écrits avant 1872 . Voir Manfred Kuhne, dans Anzengrubers Werke, op. cit., t. I, p. 352.

26. Voir Ferdinand von Saar, Novellen aus Österreicht, op. cit., t. I, p. 87-88.

27. Dans le sens de «conte de chevalerie». 
entre le Bien et le Mal : d'un côté, les ouvriers Georg Huber et sa collègue bohémienne, Tertschka; de l'autre, le surveillant cruel, beau-père de la dernière. Codé comme le contraste entre la lumière et les ténèbres, ce conflit fait penser à la lutte entre saint Georges et le dragon ${ }^{28}$. Le narrateur de l'histoire fait aussi des commentaires sur ce qui se passe, un procédé rejeté par le naturalisme zolien. Dans Die Steinklopfer, où l'accent est mis sur le quotidien des ouvriers, il est question du sort des individus, au lieu de celui d'une classe sociale comme collectivité, comme c'est le cas dans les textes naturalistes sur le prolétariat qui suivront ${ }^{29}$. Néanmoins, cette nouvelle devance le courant naturaliste de la représentation du prolétariat rural.

Die Steinklopfer affiche en effet des caractéristiques importantes du naturalisme, et non seulement dans le choix de personnages marginalisés et de la classe ouvrière, mais aussi à cause de la contemporanéité de son sujet et de la description de la dure réalité du chantier de construction, avec toutes ses injustices. Le surveillant impitoyable organise le travail et l'hébergement des ouvriers en tournant tout à son avantage. La nouvelle traite aussi d'autres thèmes naturalistes moins usuels, comme de l'abus sexuel. Terschka explique à Georg sa situation comme belle-fille du surveillant qui avait essayé de la violer :

Schon zur Zeit, da die Mutter noch lebte, wollte er oft zärtlich mit mir tun, aber ich wich ihm aus und drohte, ich würd' es der Mutter klagen. Im vorigen Sommer jedoch kam er eines Abends allein aus dem Wirsthaus zurück und fing wieder an und sagte, er würde mich heiraten. Und da ich ihm kein Gehör gab, wollt' er Gewalt brauchen. Ich aber hab' mich seiner erwehrt und hab' ihm gesagt, was ich von ihm denke. Seitdem haßt er mich bis aufs Blut und rächt sich, wie er kann ${ }^{30}$.

Une telle représentation réaliste du côté sombre du quotidien pénible des ouvriers anticipe la constitution officielle de l'école naturaliste d'Émile Zola, à un moment où la transgression des bienséances bourgeoises dans ses romans urbains produisait des succès à scandale ${ }^{31}$.

28. Rainer Baasner, art. cité, p. 55, 57, 64 et 66 .

29. Voir Herbert Klauser, op. cit., p. 103.

30. Ferdinand von Saar, Novellen aus Österreicht, op. cit., t. I, p. 112 : «Déjà, à l'époque où maman était encore en vie, il se montrait souvent affectueux à mon égard, mais j'essayais toujours d'échapper à ses avances et le menaçais de me plaindre à ma mère. Mais l'été dernier, il est rentré seul de l'estaminet et a recommencé, et il a dit qu'il m'épouserait. Comme je ne voulais point l'écouter, il a voulu employer la force. Je me suis défendue, d'ailleurs, et je lui ai dit ce que je pensais de lui. Depuis, il me hait et essaie de se venger à chaque occasion.»

31. David Baguley, op. cit., p. 12 et 176. 
Une autre dimension des Steinklopfer fait voir des embellies, comme l'idylle de vie champêtre que le couple entrevoit le dimanche quand il s'échappe et se rend au marché de Schottwien et à l'église Maria Schutz, avec des scènes mémorables, comme cette fête de mariage que les casseurs de pierre misérables observent de loin, n'osant pas s'imaginer une telle possibilité. En dépit des circonstances affreuses qui mènent à une altercation tendue et violente entre Georg et le surveillant, tout ira bien pour le couple. Figure de deus ex machina, c'est le colonel (der Oberst) qui doit décider du sort de Georg, un soldat en permission spéciale (Urlauber), maintenant accusé d'avoir tué le surveillant. Après avoir compris l'amour de Tertschka pour le prisonnier, le colonel décide de disculper Georg du crime de meurtre. Il joue un rôle clé, car c'est lui qui doit nommer quelqu'un au poste de garde-barrière, qui occuperait la petite maison construite des décombres qui restaient sur le chantier, à côté de la voie ferrée. La dernière image de Georg et Terschka devant leur maison rappelle certes le happy end des contes de fée. Ils embrassent leurs enfants aux cheveux blond filasse, en regardant le soleil qui se couche:

Auch trauliche Abendstunden sind ihnen vergönnt, wo sie Hand in Hand vor der Türe sitzen, der untergehenden Sonne nachschauen und noch immer den Tag preisen, an welchem sie sich zum ersten Male auf der Höhe des Semmerings begegnet. [...] Und wenn dann in die Helle ihrer Brust ein trüber, dunkler Schatten fallen will, dann rufen sie schnell die Kleinen heran, die sich liebkosend in die Arme der Eltern schmiegen und mit den großen Kinderaugen so harmlos in die Welt hineinblicken, als lebten sie nicht den wechselvollen Schicksalen entgegen, die sich forterben von Geschlecht zu Geschlecht, so lange noch Menschen atmen auf der alternden Erde $e^{32}$.

La vision entropique du naturalisme semble manquer ici, car l'action est suspendue au moment du bonheur dont jouissent les misérables, sur fond de déclin général. Dans un texte naturaliste comme La Terre, la dégénérescence domine et continue ${ }^{33}$, tandis que, dans Die Steinklopfer,

32. Ferdinand von Saar, Novellen aus Österreicht, op. cit., t. I, p. 122-123: «Ils jouirent aussi d'heures harmonieuses, pendant lesquelles, assis devant leur porte, main dans la main et regardant le coucher du soleil, ils remerciaient toujours le jour où ils se rencontrèrent pour la première fois, sur les hauteurs du Semmering. [...] Quand, dans la lumière de leur joie, une ombre triste et sombre menace de tomber, ils appellent vite les enfants qui viennent se presser affectueusement dans les bras des parents et, avec leurs gros yeux enfantins, regardent le monde de façon si inoffensive, comme s'ils ne s'apprêtaient pas à vivre l'expérience des destins changeants dont ils allaient hériter à leur tour et qui passent de génération à génération, aussi longtemps que les hommes respirent sur une terre vieillissante.»

33. David Baguley, op. cit., p. 208. 
le narrateur termine son histoire au moment où les protagonistes voient la réalisation de leur rêve, malgré tout ce qui l'avait contrecarré. Tertschka et Georg font aussi montre d'un sens éthique élevé et ils ont l'espoir de mieux vivre que les autres ouvriers, qui passent leur temps libre à boire et à jouer aux cartes ${ }^{34}$. La pureté de leur amour et leur sens de la justice rendent possible leur ascension au-dessus de la collectivité ouvrière, surtout par contraste avec les paysans libres dans La Terre de Zola, où la population rurale semble entièrement obsédée par la possession de la terre, une cupidité insatiable qui se confond souvent avec la conquête sexuelle.

Une autre nouvelle de Saar, Die Troglodytin, diffère de Die Steinklopfer, d'abord par la situation narrative, le personnage principal racontant sa propre histoire. Dans le récit-cadre, Pernett, maintenant vieillard, se souvient de son premier emploi comme forestier, subordonné du vieil officier des Forêts pour la région (Revierförster), au service d'un propriétaire noble morave - un comte. Ce type de narration permet de mettre en valeur le visuel, le narrateur se rappelant et évoquant, des décennies plus tard, ses rencontres érotiques avec le personnage principal féminin, Maruschka. C'était la fille d'un ivrogne dont la famille avait emménagé, au moins partiellement, dans une sorte de grotte qui restait d'un campement de gitans nomades - d'où le titre de la nouvelle. L'accent est mis sur ces échanges à la fois stimulants et malsains, qui frisent le voyeurisme, avec le compte rendu du personnage-narrateur des attitudes de Maruschka, souvent légèrement vêtue de chiffons ${ }^{35}$. La description détaillée étant une caractéristique capitale de la fiction naturaliste, la fascination du corps féminin prend ici une signification particulière.

Certains passages dans Die Troglodytin, comme dans d'autres nouvelles de Saar, créent des scènes pittoresques, comme celle où des femmes courbées, comme des fleurs géantes, avec leurs foulards aux couleurs vives qui flottent dans la brise, sarclent de jeunes plantes de navet vert émeraude qui font contraste avec les champs de blé jaunissant. Cette dernière image suggère que la fin de la vie des plantes approche, dans

34. Tertschka explique à Georg pourquoi elle veut se rendre à Schottwien, et aller à l'église: "Ich mag nicht das Beten ganz verlernen unter dem Volk, das nur ans Trinken und Kartenspielen denkt » (Ferdinand von Saar, Novellen aus Österreicht, op. cit., t. I, p. 103).

35. Karl Wagner, art. cité, p. 226. 
un paysage autrement très vivant. Comme le note Baguley, une poétique de la dissolution marque, dans un texte naturaliste, même les passages qui semblent être des descriptions les plus innocentes ${ }^{36}$.

D'autres références au paysage ou à l'atmosphère soulignent le caractère potentiellement transgressif de l'attraction sexuelle, qui est le thème principal de la nouvelle, et ces références s'alignent avec la construction de l'intrigue. Un jour d'été, la chaleur du soleil pousse Pernett, pendant sa randonnée de surveillance, dans l'ombre de la forêt à la recherche d'une source où il peut étancher sa soif. Que trouve-t-il à l'endroit même où il sait qu'il y a de l'eau? Maruschka, étendue sur l'herbe dans une pose séduisante, après avoir ôté tous ses vêtements. Elle jouit de l'ombre et du son de l'eau qui coule. Tout d'abord, en prenant conscience de la présence de Pernett, elle lève la main aux yeux; puis, elle compose l'attitude stéréotypée de Vénus, bien qu'elle n'ait jamais vu ni statue ni peinture de Vénus de sa vie, remarque le narrateur ${ }^{37}$.

Le style narratif, fonctionnant principalement par ces comptes rendus entichés du voyeurisme, renforce le thème du désir sexuel. Dans le roman naturaliste, la nature (souvent figurée par une femme) agit directement sur le sujet humain, ce qui occasionne ruine et destruction. Comme le remarque Baguley, la femme, qui représente le biologique capable de détruire le social, sert souvent d'instigatrice ou figure de la désagrégation ${ }^{38}$. Ici, dans la nouvelle de Saar, le désir sexuel est toujours en conflit avec les efforts de Pernett de rester maitre de la situation. Il a pour tâche de surveiller et de gérer la forêt et les espaces qui la côtoient et de communiquer toute irrégularité au Revierförster. Avouant son faible pour le sexe féminin et conscient du fait qu'il se trouve

36. Je paraphrase, en français, le passage suivant: «Wie Smaragd stachen die Rübenpflanzungen von dem gelblich wogenden Getreide ab, und die bunten Kopftücher der Arbeiterinnen, die sehr zahlreich in tief gebückter Haltung auf dem grünen Plan beschäftigt waren, flatterten wie seltsame große Blumen (Ferdinand von Saar, Novellen aus Österreicht, op. cit., t. II, p. 119). En ce qui concerne «th[e] poetics of dissolution ", voir David Baguley, op. cit., p. 200.

37. Je paraphrase, en français, le passage suivant : « sofort aber zuckte es eigentümlich um ihren Mund, und indem sie die erhobenen Arme sinken ließ, nahm sie die bekannte Venusstellung an, die sie nie im Leben vor Augen gehabt" (Ferdinand von Saar, Novellen aus Österreicht, op. cit., t. II, p. 114).

38. David Baguley, op. cit., p. 210. L'auteur cite Patrick Wald Lasowski, qui écrit, au sujet de Nana de Zola : "Nana corruptrice, [...] en qui s'incarne le mal, c'est aussi le Peuple, la Nature et la Femme, forces effrayantes, fascinantes et incontrôlées [...]» (ibid., p. 211). 
entouré de "willfährige Sklavinnen ${ }^{39}$ ", le forestier-narrateur raconte les événements et exprime ses soucis. Comme subordonné fidèle, il essaie d'accomplir les tâches de son service et de maintenir son statut comme membre respecté de la communautét ${ }^{40}$.

Vivant et agissant en dehors des normes de la société, les membres de la famille Kratochwil deviennent les plus difficiles de tous les faiseurs d'histoires de la région et méritent ainsi d'être l'objectif principal des enquêtes et des pérégrinations de Pernett. Son regard vise la fille fascinante, Maruschka, qui trouve le forestier également attrayant. Elle profite de toute occasion pour se placer sur son chemin, s'offrant, et non seulement comme employée, quand il lui suggère de trouver du travail dans l'agriculture: "Nehmt Ihr mich lieber. [...] Ihr könnt mich ja auch im Walde brauchen ${ }^{41}$."

Avec l'accent mis sur les traits séduisants d'une jeune paria entrainnant potentiellement des conséquences dangereuses, les structures de la société rurale et les changements contemporains figurent de façon révélatrice dans cette nouvelle. L'objectif de son projet étant de faire un portrait de la vie dans l'empire d'Autriche ${ }^{42}$, Saar rend compte de l'organisation sociale pendant cette période et dépasse dans son analyse de la situation rurale les naturalistes ${ }^{43}$. Le vieux Revierförster remarque que maintenant, avec l'arrivée de l'industrialisation dans les campagnes (ce qui, avant 1866, était le cas surtout en Bohême et en Moravie), les indigènes sont plus ou moins responsables de leur propre bien-être, une situation qui les éloigne en principe d'une organisation féodale. À l'époque de ces événements, il y a une usine sidérurgique dans la

39. Ferdinand von Saar, Novellen aus Österreicht, op. cit., t. II, p. 102 : «des esclaves dociles.» Eugen Thurnher, dans «Soziale Problematik im Werk von Ferdinand von Saar» (Karl Konrad Polheim dir., Ferdinand von Saar. Ein Wegbereiter der literarischen Moderne. Festschrift zum 150. Geburtstag, Bonn, Bouvier, 1985, p. 45), appelle les nouveaux employés de la révolution industrielle "Arbeitssklaven».

40. Dans l'ébauche de Germinal, Zola écrit : «Les ouvriers lâchés vont jusqu'au crime; il faut que le lecteur bourgeois ait un frisson de terreur» (cité dans David Baguley, op. cit., p. 176).

41. Ferdinand von Saar, Novellen aus Österreicht, op. cit., t. II, p. 117: «Prenez-moi, plutôt. [...] Vous pouvez m'employer dans la forêt. »

42. Après avoir publié plusieurs de ces nouvelles, Saar écrit à Marie zu Hohenlohe: "Jede meiner Novellen ist ein Stück österreichischer Zeitgeschichte [...]» (Chacune de mes nouvelles est un morceau d'histoire contemporaine de l'Autriche, lettre du 8 février 1889, citée dans Ferdinand von Saar, Novellen aus Österreicht, op. cit., t. II, p. 298).

43. Eda Sagarra, "Social Types and Social Reality in the Narrative Fiction of Ferdinand von Saar », dans Karl Konrad Polheim (dir.), Ferdinand von Saar..., op. cit., p. 150. 
petite ville, qui est un centre administratif de moindre importance, abritant néanmoins une cour de justice et ayant son propre maire - le Bürgermeister, un propriétaire terrien et chef du parti politique "slave». Quelques artisans indépendants de la région habitent dans un village industriel modèle d'inspiration anglaise, établi par la génération précédente. Sur la rive fissurée de la rivière, une colonie de familles ouvrières et d'hommes à tout faire s'étaient installée, là où le père de Maruschka avait sa hutte (Kaluppe) quand il a connu des jours meilleurs. Les femmes et les filles de ces hommes font des journées dans les champs aux alentours. Souvent on permet aussi aux villageoises, sous la surveillance du forestier, de ramasser dans la forêt les branches et les brindilles qui sont tombées. Les villageois jouissent donc d'une indépendance relative, mais ce ne sont pas des paysans libres avec leurs propres terres, comme dans le roman de Zola.

La famille de Maruschka se marginalise par rapport aux autres villageois, non seulement en raison de l'ivrognerie du père, mais aussi parce que les parents choisissent de refuser le monde du travail et l'intégration à la vie de la communauté - une séparation indiquée topographiquement. Le Revierförster et Pernett sont scandalisés par la paresse et le manque de responsabilité qu'affichent les parents de Maruschka - surtout en laissant leurs enfants courir et en les encourageant à mendier et à voler, au lieu de les envoyer à l'école. Si les villageois ne viennent pas à leur aide, ce sera donc à ces deux officiels de s'occuper d'eux, se plaint le Revierförster ${ }^{44}$.

La beauté aux yeux verts de Maruschka la rend attrayante à un degré inquiétant et dangereux ${ }^{45}$ pour Pernett qui, bien que d'origine slave lui-même, s'est intégré à l'administration et à la culture germanophones. Pour le Revierförster, d'ailleurs, Maruschka est le symbole de la dégénérescence, du déclin général de la paysannerie ${ }^{46}$. À un moment, Pernett décide de raisonner Maruschka et de l'encourager à s'engager comme journalière dans les champs de navets avec les autres femmes et filles, lui offrant un peu d'argent comme motivation. Peu après, Maruschka exhibe un nouveau foulard d'un rouge vif couleur de feu ( «ein feuerfarbenes Kopftuch ${ }^{47}$ »), une sorte de déclaration de sa passion pour Pernett et, en même temps, un indice métonymique de la suite de

44. Ferdinand von Saar, Novellen aus Österreicht, op. cit., t. II, p. 118.

45. En allemande : « eine echte slawische Schönheit », ibid., p. 121.

46. Ibid., p. 118-119.

47. Ibid., p. 119. 
l'histoire. Maruschka sera accusée à tort d'avoir mis le feu à la maison du garde-chasse; elle le fera pour de vrai, à son retour de prison, incendiant, par vengeance, la maison du Bürgermeister qui lui en voulait d'avoir entraîné son fils, d'esprit lent, à la suivre dans la forêt pour des relations sexuelles. Maruschka reproche au Bürgermeister, et au village entier, de l'avoir accusée faussement d'incendie criminel et de l'avoir envoyée pour la deuxième fois en prison.

Après sa libération, Pernett remarque que Maruschka ne se présente plus dans l'attitude de la Zuchthausprinzessin avec ses chiffons ${ }^{48}$, comme après sa première incarcération et les travaux forcés. Maintenant, elle manifeste clairement les effets de la dégénérescence - la tête en avant, le corps gonflé et le visage bouffi, terne et absent ${ }^{49}$. Pendant que Pernett observe, d'une position en aval, la maison en feu du Bürgermeister, il prend connaissance de la présence de Maruschka, tout près dans les ténèbres, assise sur un rosier chargé de fruits mûres. Dans la nature, comme pour Maruschka, la saison florissante s'est écoulée. Elle ne nie pas avoir incendié la maison, et Pernett remarque qu'elle adoucit maintenant sa souffrance et alimente sa colère avec du Branntwein, une boisson qui l'associe encore une fois au feu, mais également au père alcoolique - et à la tare héréditaire naturaliste. Maruschka se vante de son succès et insiste sur son dégoût du travail et de la prison. Quand Pernett lui dit qu'elle ira de nouveau en prison, et que, cette fois-ci, ça sera pour dix ans de travaux forcés, elle se moque de lui, disant qu'on ne l'attrapera jamais. Pourquoi ne s'empare-t-il pas d'elle en ce moment, lui demande-t-elle? Elle met la main sur le fusil qu'il porte, criant: «Schießt mich nieder!» Mais elle change subitement son défi : "Oder nein, küßt mich $!^{50}$ » D'abord, elle se jette par terre à ses pieds, puis elle essaie de l'entraîner dans la forêt, lui rappelant, à sa honte, qu'il avait été amoureux d'elle.

Ce qui survient se prête à une interprétation freudienne - en rapport avec ses théories de de la sexualité ${ }^{51}$. Dans la perspective de Pernettnarrateur, qui essaie de se dégager de la forte poigne de Maruschka,

48. Ibid., p. 110.

49. Ibid., p. 131.

50. Ibid., p. 135.

51. Voir, par exemple, Sigmund Freud, Trois essais sur la théorie sexuelle, Pierre Cotet et Franck Rexand-Galais (trad.), Paris, PUF, 2010, p. 89-90, où il est question du couple "plaisir-déplaisir» qui crée la tension sexuelle, une première étape menant à l'acte sexuel. 
le lecteur subit l'expérience de ses sensations confuses: le dégoût et la peur - mais, au même moment, le désir sexuel :

Und trotz allen Ekels und Abscheu, trotz der Furcht, die ich jetzt vor ihr empfand, fühlte ich eine plötzliche Wallung des Blutes, meine Sinne drohten sich zu verwirren; ich befand mich in einer entsetzlichen Lage ${ }^{52} \ldots$

Maruschka disparaît dans la forêt à la faveur de la nuit; puis, au printemps, après la fonte de la neige, on retrouve son corps déformé dans un fossé, rappelant la désintégration du corps féminin à la fin de Nana (1880) de Zola. Les thèmes de l'hérédité et du désir sexuel - ou des pulsions physiologiques - font partie du programme naturaliste, ainsi que la représentation de la figure féminine comme destructrice de l'organisation sociale.

\section{Conclusion}

Nous avons essayé de montrer que, vers le début de sa carrière de nouvelliste, Die Steinklopfer et Die Troglodytin - cette dernière tout particulièrement - révèlent que Saar fait vraiment un pas en direction de la représentation naturaliste de l'environnement rural. La situation narrative de Die Troglodytin a plus de vraisemblance que celle de Die Steinklopfer; c'est une histoire racontée de la perspective $\mathrm{du}$ personnage-narrateur qui a vécu les événements, ce qui ajoute une dimension très personnelle et permet à l'auteur de décrire le milieu - le paysage, les conditions de travail et l'organisation sociale -, de reproduire des dialogues, mais aussi de fixer de façon presque voyeuriste la figure féminine, Maruschka. Ce sont là des traits de la fiction naturaliste.

En ce qui concerne les structures plus profondes de ces nouvelles, en rapport avec la vision «entropique» du naturalisme, Die Troglodytin présente surtout des ressemblances avec le roman naturaliste zolien. La jeune Slave sert de topos principal de la désintégration dans une communauté rurale, de figure du chaos et de la menace de la sexualité féminine déchaînée. De sa position de l'extérieur, Maruschka devient symbole de tout ce qui pourrait empêcher le groupe social de fonctionner

52. Ferdinand von Saar, Novellen aus Österreicht, op. cit., t. II, p. 135 : «En dépit de tout le dégoût, de toute l'aversion, en dépit de la crainte que je ressentais maintenant devant elle, je sentis néanmoins le sang monter en moi, je fus sur le point de perdre la tête; je me trouvai dans une situation épouvantable...» 
et de se développer. Elle refuse de travailler, de s'instruire, de participer à la vie de la communauté, de se marier, de respecter la propriété d'autrui. Maruschka n'est cependant pas associée à la maladie, répandant la contagion dans la bourgeoisie, comme la figure féminine dans $\mathrm{Nana}$, dans lequel le motif de la "mouche d'or» domine; par contre, elle est associée au feu, comme symbole à la fois de la chaleur de la passion et de la menace de la destruction par une contagion moins organique que la "petite vérole» - une conflagration qui évoque, plutôt, le chaos social total de l'idéologie révolutionnaire dans Germinal (1885):

C'était la vision rouge de la révolution qui les emporterait tous, fatalement, par une soirée sanglante de cette fin de siècle. [...] Des incendies flamberaient, on ne laisserait pas debout une pierre des villes, on retournerait à la vie sauvage dans les bois ${ }^{53}$.

Il est impossible aussi de ne pas penser aux pétroleuses de la guerre franco-prussienne en 1870-1871 - encore des femmes qui semèrent la destruction et le chaos.

Ce serait peut-être le point le plus intéressant sur lequel Die Troglodytin diffère du roman naturaliste "classique " - la manière précise dont la figure féminine est utilisée comme symbole. Pernett remarque les changements que subit le corps de Maruschka tout le long de la nouvelle; ceux-là semblent survenir en rapport avec les restrictions exercées sur ses libertés, comme résultat de son emprisonnement, et non exclusivement pour des raisons héréditaires, c'est-à-dire, par la dégénérescence. Les modifications subies par le corps de Maruschka signifient peut-être d'une autre manière l'évolution des structures sociales contemporaines dans l'empire d'Autriche. Les citoyens ordinaires trouvaient toujours leur quotidien circonscrit par l'autorité du propriétaire terrien ou du capitaliste industriel, une situation qui limitait les possibilités de ceux et celles qui voulaient vivre autrement. Ce système intermédiaire semblait offrir une mesure de liberté, mais il dépendait du bon fonctionnement de la communauté, dont les membres devaient veiller sur les marginalisés et sur ceux qui souffraient. Le personnage de Maruschka pourrait donc figurer, avec son foulard rouge, la fortune ambiguë du concept de liberté. Terschka, dans Die Steinklopfer, avait également un foulard rouge, qu'elle portait le dimanche où elle avait rompu brièvement les chaînes qui la liaient au chantier et à son beau-père cruel. Tandis qu'elle réussit à échapper définitivement à sa situation d'esclavage, Maruschka, elle, ne trouve sa délivrance que dans la mort.

53. Émile Zola, Germinal, Paris, Flammarion, 1968, p. 345-346. 